\title{
Design and Implementation of a Persian Questionnaire for the Evaluation of Internal Medicine Residents' Skills in Upper Gastrointestinal Endoscopy
}

\author{
Raika Jamali ${ }^{1, *}$ \\ ${ }^{1}$ Research Development Center, Sina Hospital, Students' Scientific Research Center, Digestive Disease Research Institute, Tehran University of Medical Sciences, Tehran, IR Iran \\ "Corresponding author: Raika Jamali, Research Development Center, Sina Hospital, Students' Scientific Research Center, Digestive Disease Research Institute, Tehran \\ University of Medical Sciences, Tehran, IR Iran. Tel: +98-2122912240, E-mail: raika.jamali@gmail.com
}

Received 2016 March 12; Revised 2016 March 19; Accepted 2016 March 26.

Keywords: Surveys and Questionnaires, Clinical Competence, Endoscopy, Education

It seems reasonable to define residents' pitfalls during their attendance of a diagnostic upper gastrointestinal (GI) training course. In this regard, an assessment tool is necessary to evaluate these skills. Although various questionnaires are currently used worldwide for this purpose, a valid Persian version is lacking. The aim of this project was to develop a valid Persian questionnaire to accurately assess residents' skills while performing upper gastrointestinal endoscopy.

First, a review of the literature was performed in the databases of web of science, PubMed, CINAHL, Embase, Scopus, and Google Scholar using the following keywords: "endoscopy", "training”, "resident”, and "assessment". All articles and abstracts in English through 2014 were reviewed. The questionnaire was designed based on the aims of endoscopy training courses in internationally well-known GI educational centers. After the review, 24 items were recognized as important in the assessment of trainees. For the next step, 14 items were excluded due to their weak ability to evaluate the aims of the training course, based on the expert opinion of GI specialists at Tehran University of Medical Sciences, who assessed the content validity of the questionnaire. The faults of the questionnaire were revised in the pilot study. The final questionnaire contained 10 items with scores of 1-3 based on a Likert scale. The trainer filled out the questionnaire, and the result was provided to the trainee at the end of procedure. The reliability and validity of the developed questionnaire was checked by evaluating 20 internal medicine residents at the end of their training course between 2014 and 2016.

The calculated Cronbach's alpha (reliability coefficient) range was between 0.79 and 0.94 for the different domains, indicating a high level of internal consistency for the questionnaire. The measured intra-class correlation range was between 0.66 and 0.90 for the 10 items, which showed a good level of inter-rater reliability.

The results showed that the newly developed Persianlanguage questionnaire was appropriately accurate with regard to determining residents' skills in GI endoscopy. 